\title{
Inhalt, Vol. 13, No. 5, 1993
}

\section{Contents}

Übersichtsarbeiten

Aktiv-spezifische Immuntherapie - Eine neue Modalität in der Krebsbehandlung, die das patienteneigene Immunsystem mit einbezieht

Schirrmacher, V. 290

Die Bedeutung von Tamoxifen in der Prevention des Mammakarzinoms

Götzinger, P., Gnant, M., Hochstöger, E., Jakesz, R 297

Laufende und neue Konzepte bei der systemischen adjuvanten Behandlung des

Mammakarzinoms

Kaufmann, M 304

Einsatz von G-CSF und GM-CSF zur Unterstützung chemotherapeutischer Verfahren im

klinischen Alltag: Perspektiven und Herausforderungen

Greil, R $\quad 310$

Taxol: Eine neue antineoplastische Substanz

Diergarten, K., Dreps, A 329

Review Articles

Active Specific Immunotherapy - A New Modality of Cancer Treatment Involving the Patient's

Own Immune System

Schirrmacher, V. 290

The Potential Impact of Tamoxifen in Breast Cancer Prevention

Götzinger, P., Gnant, M., Hochstöger, E., Jakesz, R 297

Current and New Concepts in the Systemic Adjuvant Treatment of Breast Cancer

Kaufmann, M 304

The Application of G-CSF and GM-CSF as an Adjunct to Chemotherapy in Clinical Practice:

Perspectives and Challenges

Greil, R 310

Taxol: A New Antineoplastic Agent

Diergarten, K., Dreps, A 329

Originalarbeiten

Malignome nach Herztransplantation

Stauch, C, Fischer, B., Bernhard, A

Original Paper

Malignancies after Heart Transplantation

338 Stauch, C, Fischer, B., Bernhard, A

338 
Zugehörigkeit zu einer prognostischen Untergruppe als wichtigstes Kriterium für den Krankheitsverlauf bei metastasiertem Mammakarzinom: Ergebnisse einer dreiarmigrandomisierten multizentrischen Studie zum Vergleich von Doxorubicin, Epirubicin und Mitoxantron jeweils in Kombination mit Cyclophosphamid Heidemann, E., Steinke, B., Hartlapp, J., Schumacher, K., Possinger, K., Kunz, S., Neeser, E., v. Ingersleben, G., Hossfeld, D., Caffier, H., Souchon, R., Waldmann, R., Blümner, E., Clark, J. ... 344

Chronologische Veränderungen beim Magenfrühkarzinom

Reitzig,P,Brockmann,B., Gütz, H.-J,, Wildner, G.P. 354

Prognostic Subgroups: The Key Factor for Treatment Outcome in Metastatic Breast Cancer:

Results of a Three-Arm Randomized Multicenter Trial Comparing Doxorubicin, Epirubicin and Mitoxantrone Each in Combination with Cyclophosphamïde

Heidemann, E., Steinke, B., Hartlapp, J., Schumacher, K., Possinger, K., Kunz, S., Neeser, E., v. Ingersleben, G., Hossfeld, D., Caffier, H., Souchon, R., Waldmann, R., Blümner, E., Clark, J. ... 344

Chronological Changes in Early Gastric Cancer

354

Reitzig, P., Brockmann, B., Gütz, H.-J., Wildner, G.P.

Kurzmitteilung

Intraperitoneale Chemotherapie mit Carboplatin in der Behandlung des fortgeschrittenen

Ovarialkarzinoms: Erfahrungsbericht in zwei Fallen

Schwella, N., Riess, H, Lübbe, A.S., Salama, Z.B., Huhn, D. .

360

Short Communication

Intraperitoneal Carboplatin in the Treatment of Bulky Disease Ovarian Cancer: Experience in Two Cases

Schwella, N., Riess, H., Lübbe, A.S., Salama, Z.B., Huhn, D.

i60

Fortsetzung aufSeite 288

Continued on page 288

Band 16, Heft 5, Oktober 1993

Internationale Zeitschrift für Krebsforschung und -behandlung

Inhalt

Contents

Klinische Information

Brusterhaltende Therapie und Wiederaufbauplastik beíin Mammakarzinom

Lemperle, G., Exner, K

Clinical Information

Breast-Preserving Therapy and Breast Reconstruction after Carcinoma

364 Lemperle, G, Exner, K

364 
Der Krebsinformationsdienst (KID) am Tumorzentrum Heidelberg/Mannheim: Aktuelle onkologische Information für die Öffentlichkeit

Humbert, K.-D 371

Cancer Information Service (KID), Tumor Center Heidelberg/Mannheim: Current Oncological Information for the Public

Humbert, K.-D 371

Buchbesprechung 377 Book Review

377

Mitteilungen onkologischer Gesellschaften

Mitteilungen der Arbeitsgemeinschaft Internistische

Onkologie (AIO)

Reports of Oncological Societies

Reports of the Arbeitsgemeinschaft Internistische

380 Onkologie (AIO) 380

Impressum 285

Inhaltsverzeichnis 286

Industrieforum 378

Hinweise für Autoren 383

Imprint285

Contents 286

Industrial Forum $\quad 378$

Instructions to Authors 383

Bibliographischer Hinweis: Inhaltsverzeichnisse dieser Zeitschrift erscheinen regelmäßig in current contents ${ }^{\circledR}$ sowie in anderen bibliographischen Diensten. 